\title{
Pattern-contingent color aftereffects on noninduced patterns
}

\author{
G. KEITH HUMPHREY \\ University of Western Ontario, London, Ontario, Canada \\ PETER C. DODWELL \\ Queen's University, Kingston, Ontario, Canada \\ and \\ VICTOR F. EMERSON \\ Bell-Northern Research, Ottawa, Ontario, Canada
}

\begin{abstract}
In a series of experiments, we found that in addition to expected reports of color aftereffects on patterns viewed during induction, reliable and predictable reports of color were given by subjects to patterns they did not view during induction. These reports to noninduced patterns were generally to patterns that were orthogonal to the patterns seen during induction. Induction with, for example, a red vertical grating led to appropriate aftereffects (i.e., green) on that vertical pattern and to the complementary aftereffect (i.e., pink) on a horizontal grating. We suggest that such color aftereffects on noninduced patterns are based on a shift in the activity of orientation coding mechanisms as a result of viewing the inducing patterns. We further propose that the results are consistent with the Lie transformation group theory of neuropsychology and that they add to a growing body of research demonstrating the applicability of this theory to the understanding of pattern-contingent color aftereffects.
\end{abstract}

Pattern-contingent color aftereffects (PCCAEs), or McCollough effects, were first reported over 20 years ago (McCollough, 1965). Such effects are induced by viewing, for example, a black-and-white vertical grating in red light alternating with a black-and-white horizontal grating in green light for an induction period lasting several minutes. Following such inspection, achromatic gratings in white light appear to be tinted with weakly saturated hues that are approximately complementary to those paired with each orientation during initial viewing. Although a great deal of effort has been devoted to understanding the mechanisms underlying such effects (for reviews, see Harris, 1980; Stromeyer, 1978), there is still strong disagreement about how or why these effects are generated (Skowbo, 1986).

In a series of recently published experiments (Dodwell \& O'Shea, 1987; Emerson, Humphrey, \& Dodwell, 1985; Humphrey, Dodwell, \& Emerson, 1985), we have shown that at least some PCCAEs must be generated at a level of processing in the visual system that is beyond the simple fatiguing of individual color- and orientation-sensitive units, the traditional neuropsychological postulate for ex-

This work was supported by Natural Sciences and Engineering Research Council of Canada Grant A1643 to G. K. Humphrey and Grant A0044 to P. C. Dodwell and D. W. Muir. Thanks to Bonnie Williams for data collection. Thanks to one anonymous referee for many helpful suggestions. Address requests for reprints to G. Keith Humphrey, Department of Psychology, University of Western Ontario, London, Ontario N6A 5C2, Canada. plaining the effects. We also sought to demonstrate the applicability of the Lie transformation group theory of neuropsychology (LTG/NP), originally developed by Hoffman (1966), to the understanding of PCCAEs. The vectorfield structure and modus operandi of LTG/NP seem well-suited to be candidates for the processing of PCCAEs. Three basic pattern pairs are derived from the transformation orbits of the "primitive model" of LTG/NP (Dodwell, 1983): horizontal and vertical gratings, concentric circles and radial lines, and $90^{\circ}$ and $45^{\circ}$ oriented rectangular hyperbolas. They all show high degrees of symmetry, and the patterns in a pair are locally and globally orthogonal. Our experiments have demonstrated that the basic pattern pairs of LTG/NP can support independent PCCAEs (Emerson et al., 1985) and that the aftereffects seem to depend on the global properties or coherence of the patterns (Dodwell \& O'Shea, 1987). Such results suggest that LTG/NP provides a useful framework for understanding the generation of McCollough effects.

The orthogonal nature of the basic pattern pairs is a fundamental property of LTG/NP (Dodwell, 1983; Hoffman \& Dodwell, 1985). If superimposed on each other, the contours in one pattern would cut those of the second member of the pair at right angles at every point. Orthogonality of these patterns is a natural consequence of the orthogonal nature of the "Lie derivatives" that generate the transformations that are basic to LTG/NP (Hoffman \& Dodwell, 1985). Technically, the three transformation pairs of the "primitive model" of LTG/NP are a suffi- 
cient basis for pattern processing (Hoffman, 1978). We postulate that pattern "channels," defined and bounded by the orthogonal pairmates of the primitive model constitute the basis for pattern recognition and are the seat of generation of most (but not necessarily all) PCCAEs.

A finding of Humphrey et al. (1985, Experiment 2, Group 2.3) is especially relevant to the notion of orthogonally organized channels. Observers were induced with various combinations of the patterns shown in Figure 1. In addition to the expected reports of color aftereffects on patterns used during induction, Humphrey et al. (1985) obtained some reports of color to patterns on which the observers had not been induced. A number of observers, after induction with a pair of nonorthogonal patterns in lights of complementary color (e.g., a red bull's-eye and a green horizontal grating), reported aftereffects not only on achromatic test patterns composed of segments from the inducing patterns (i.e., bull's-eye [reported green] and horizontal lines [reported red]), but also on test patterns composed of segments from the noninduced orthogonal pairmates of the inducing patterns. That is to say, some observers reported seeing red radial segments and green vertical lines. Although the number of such reports was not great (15 out of a possible 64 reports, or $23.4 \%$ ), they were uniform. In every case, the color reported on a noninduced pattern was similar to the color in which its orthogonal pairmate had appeared during induction. Thus it appeared that induction with one member of an orthogonal pair tends to induce the com-
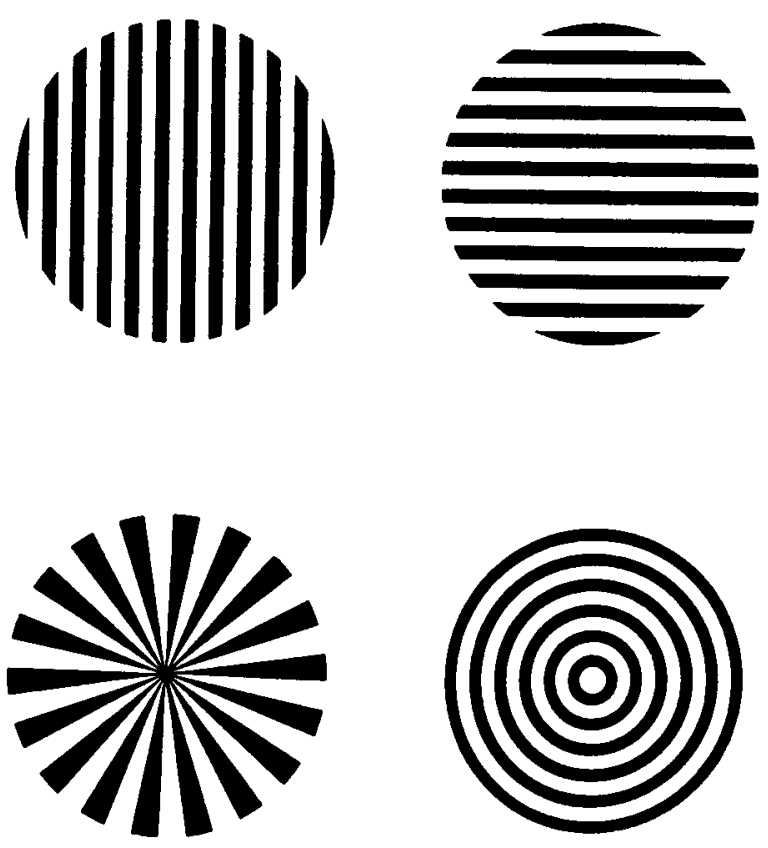

Figure 1. The patterns used during induction in the experiments. The vertical and horizontal gratings are orthogonal, as are the radial lines (star) pattern and the concentric circles (bull's-eye) pattern. The gratings were used in all of the experiments, but the star and bull's-eye were used only in Experiments 1 and 3. plementary aftereffect in its pairmate, even if the latter was not presented during induction.

The PCCAE reports on noninduced patterns obtained by Humphrey et al. (1985) are consistent with results reported by Stromeyer (1969). Stromeyer found that induction with, for example, a red vertical grating alternating with an achromatic horizontal grating produced reliable green and red aftereffects on the respective orientations. Such aftereffects could also be produced by presenting observers with a red vertical grating for 10 50 -sec periods alternating with $10 \mathrm{sec}$ of darkness. Observers induced with the latter procedure would report greenish aftereffects on achromatic vertical grating segments and reddish aftereffects on horizontal segments.

The present experiments were conducted to replicate the unanticipated result of Humphrey et al. (1985) and to explore further conditions under which induction of a color aftereffect in one pattern induces a complementary aftereffect in an orthogonal pattern. Experiment 1 involved a replication of the stimulus conditions of Humphrey et al. (1985, Experiment 2, Group 2.3) in which nonorthogonal patterns, such as the vertical grating and bull's-eye shown in Figure 1, were presented to observers in red and green light. After induction, aftereffect reports were solicited both with test patterns composed of segments of the induction patterns and with other patterns using segments from noninduced patterns.

\section{EXPERIMENT 1}

\section{Method}

Subjects. Thirty-two undergraduates, naive with respect to the purpose of the experiment, served as subjects. All had normal or corrected-to-normal vision and no known color vision deficiency. They were assigned randomly to the various induction conditions necessary for counterbalancing the possible color-pattern pairings, with 4 subjects in each pairing. Two additional subjects reported seeing colors on the pretest and were not included.

Apparatus and Stimuli. The inducing stimuli, shown in Figure 1, consisted of two pairs of orthogonal patterns: vertical and horizontal square-wave gratings, and concentric circles (bull's-eye) and radial sectors (star). The patterns were photographed on highcontrast copy film. Each was mounted in a slide holder with a Kodak Wratten 92 (red) or 74 (green) filter and projected from a Kodak Ektagraphic (III B) slide projector fitted with a Kodak Ektanar C Zoom Lens. (For convenience, these colors are referred to as complementary, although they are not strictly so.) An Apple IIe computer, interfaced with two Gerbrands tachistoscopic shutters (G1166), controlled presentation time. The patterns, which were black except for the colored bars, were rear-projected. Each pattern subtended $8.75^{\circ}$ of visual angle on the screen at the viewing distance of $130 \mathrm{~cm}$.

Separate high-contrast matte photographs of each of the patterns shown in Figure 2 were used during testing. Each pattern consisted of alternating quadrants from one pair of inducing patterns.

Design. Four possible combinations of nonorthogonal patterns were used during induction: vertical grating and bull's-eye, vertical grating and star, horizontal grating and bull's-eye, and horizontal grating and star. For each of these combinations, half of the subjects received one pattern in red and the other in green during induction, and the remaining half of the subjects received the opposite pattern-color pairing. 

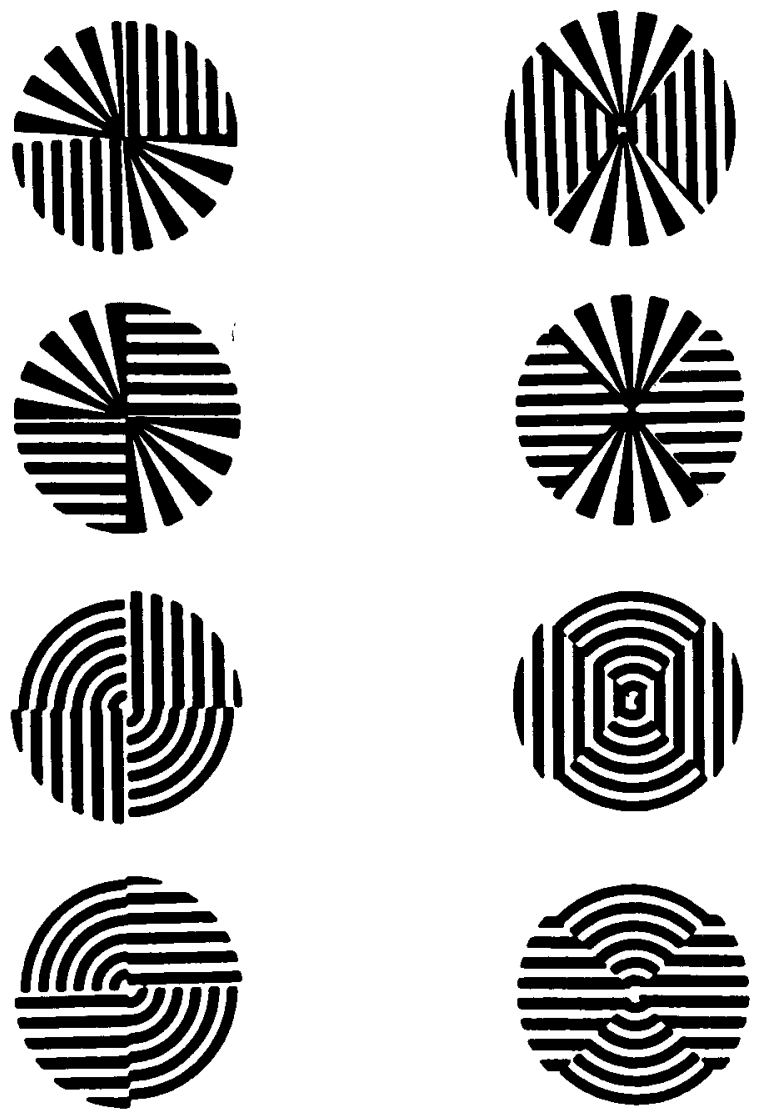

Figure 2. The eight test patterns used in Experiment 1. All of the test patterns were composed of alternating quadrants of nonorthogonal patterns.

Procedure. Each subject was tested individually in a three-stage procedure: pretest, induction session, and posttest. First, each subject was shown four of the achromatic test patterns of Figure 2. Two of the test patterns were composed of the patterns the subject would be shown during induction. The other two test patterns were composed of patterns the subject would not see during induction, but which were orthogonal to the inducing patterns. If, during the pretest, no colors were reported on any test patterns, a 20-min induction session was given. During induction, two patterns were presented alternately for $10 \mathrm{sec}$ each, with an intervening 1-sec blank period. The subject was asked to keep his/her head in a fixed position and to maintain fixation at the center of the presented pattern. Induction occurred in an otherwise dark room. The induction session was followed by a 5 -min rest period in normal room light. After this rest period, the posttest was given.

During the posttest, the subject was given a pen and a booklet containing photocopies of the four test patterns, one pattern per page. The subject was shown the test patterns in succession by an experimenter who held high-contrast copies of the patterns in front of the subject, who was instructed to fixate the center of each pattern in turn. The test patterns subtended the same visual angle as had the inducing patterns. The subject was told to report any reddish (or pinkish) or greenish areas only if both quadrants of a pattern seemed to be totally filled with the color; otherwise, he/she was to report no color. This scheme is similar to the reporting of "complete" aftereffects used in our earlier research (Emerson et al., 1985; Humphrey et al., 1985). The subject indicated the color seen by writing down the name of the color beside the appropriate part of the test pattern in their booklet.

\section{Results}

If the induction phase had been effective, negative aftereffects should have occurred to test pattern quadrants corresponding to induction pattern segments. Furthermore, if the results of Humphrey et al. (1985) were replicated, there should have been a consistent relation between the color of the induction patterns and the color reported on noninduced patterns. The color reported on a quadrant of a noninduced pattern should have been the same as that paired with its orthogonal patternmate during induction. Any color reports contrary to these expectations were considered inappropriate responses; these inappropriate reports were not ignored, but were subtracted from the number of appropriate responses for that subject. Only 4 such reports ( $1.5 \%$ of the total possible) occurred: 2 to noninduced patterns and 2 to induced patterns. (For each test pattern, 2 appropriate reports were possible. Each of the 32 subjects saw 4 test patterns, yielding a total of 256 possible reports.)

The number of appropriate reports, corrected as described above, is shown in Figure 3 for each of the patterns. It is clear that induced patterns gave rise to a high level of appropriate reports (77\% overall), whereas noninduced patterns gave rise to a lower, but still substantial, percentage of appropriate reports (59\% overall). In both cases, random assignment of color to pattern would result in a corrected frequency of zero aftereffects.

\section{Discussion}

The results confirm that PCCAEs can be established readily for each of the patterns shown in Figure 1.

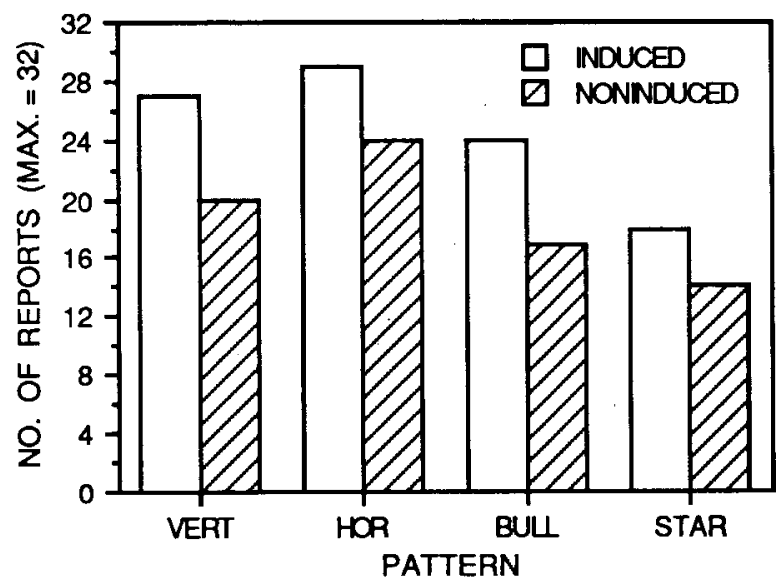

Figure 3. The results of Experiment 1 expressed as the number of pattern-contingent color aftereffects reported as a function of pattern and whether the pattern was seen during induction or not. Reports on induced patterns refer to reports on patterns seen during induction, whereas reports on noninduced patterns refer to reports on patterns not presented during induction. VERT $=$ vertical grating. HOR = horizontal grating. BULL = bull's-eye. STAR = star pattern. 
PCCAEs were reliably reported not only for induction patterns, but also for noninduced patterns whose orthogonal pairmates had been used as induction patterns; in other words, appropriate manipulation of experimental conditions reliably led subjects to report colors on patterns not seen during the induction phase. These results replicate Humphrey et al.'s (1985) similar results, which were the unexpected outcome of a control condition in another experiment. Humphrey et al. (1985) found, in the condition (Experiment 2, Group 2.3) that is comparable to the present experiment, that $40.6 \%$ and $23.4 \%$ of the possible number of appropriate reports were given to induced and noninduced patterns, respectively. The comparable values for the present experiment are $77 \%$ and $59 \%$. Although there are a number of minor differences in procedure between the two experiments, three differences stand out as likely contributors to the relatively large discrepancy in the results. First, the induction session was $20 \mathrm{~min}$ in the present experiment, but only $10 \mathrm{~min}$ in Humphrey et al. (1985). Second, color names were suggested in the present experiment, but not in the earlier one. Third, Humphrey et al. (1985) used a procedure in which subjects reported colors seen on the test patterns by outlining, as accurately as possible, the appropriate "colored" area on the corresponding pattern in the booklet. In the present experiment, subjects reported only on quadrants that seemed fully colored. These three differences could increase the number of reports in the present experiment by establishing a more vivid PCCAE through a longer induction session, by perhaps lowering subjects' criterion for report by supplying color names, and by omitting the necessity for subjects to outline the precise area in which color is seen.

The percentages of correct reports are different in the two experiments, but they are fundamentally in agreement. Achromatic test versions of noninduced patterns display the PCCAE complementary to that generated by its orthogonal patternmate. The explanation of this result is not unequivocal for the bull's-eye and star patterns, which contain multiple orientations. Consider a subject induced with a red vertical grating and a green concentric circle pattern; reports of red or pink on the noninduced horizontal grating could be the result of some orthogonally based pattern-processing mechanism such as that postulated by LTG/NP, or it could be a partial effect of induction with the concentric circles, which have a "horizontal" component. The red aftereffect reported on the noninduced horizontal grating could be the result of the color's spreading from the horizontal parts of the induced concentric circle pattern. Such an aftereffect could, by way of simultaneous color contrast, provoke reports of aftereffects on the other quadrants of the test patterns (e.g., green report on star segments).

Experiments 2, 3, and 4 were conducted to examine these alternative hypotheses. Experiment 2 again used two patterns, but each pattern represented only one major orientation. For example, the subjects were induced with colored vertical and right oblique gratings and tested with patterns composed of segments of these same gratings and with orthogonal gratings (e.g., horizontal and left oblique gratings). If aftereffect reports on the noninduced patterns in Experiment 1 were the result of spread of effect from portions of the bull's-eye and star, one would not expect aftereffect reports on noninduced patterns in Experiment 2 , since no similarly oriented components are present in any pair of patterns.

\section{EXPERIMENT 2}

\section{Method}

Subjects. Sixteen undergraduates, naive with respect to the purpose of the experiment, served as subjects. All had normal or corrected-to-normal vision and no known color vision deficiency. They were assigned randomly to the various induction conditions necessary for counterbalancing the possible color-pattern pairings, with 2 subjects in each pairing.

Apparatus and Procedure. The inducing stimuli are shown in Figure 4. During induction, the subjects were presented with two nonorthogonal patterns, such as a green vertical grating alternating with a red right oblique grating. The test patterns, examples of which are shown in Figure 5, consisted of alternating quadrants of nonorthogonal patterns. The three-stage procedure of Experiment 1 was used.

\section{Results}

It was expected that, as in Experiment 1, negative PCCAEs would be reported to parts of the test figures comprised of the inducing patterns. It also was expected that opposite PCCAEs would be reported on the noninduced orthogonal orientations, but not on the noninduced orientations at $45^{\circ}$ to the inducing orientation. "Appropriate" is defined in terms of these expectations. The number of appropriate reports for each of the patterns is shown in Figure 6. There was a high level of appropriate responding to both the induced and noninduced patterns.
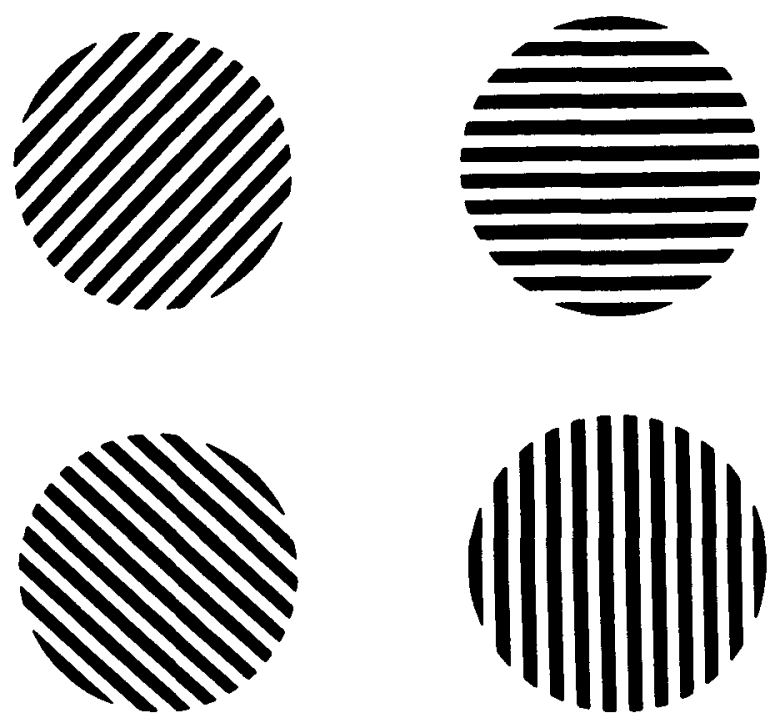

Figure 4. The patterns used during induction in Experiment 2. During induction subjects saw a colored vertical or horizontal grating paired with one of the oblique patterns in a complementary color. 

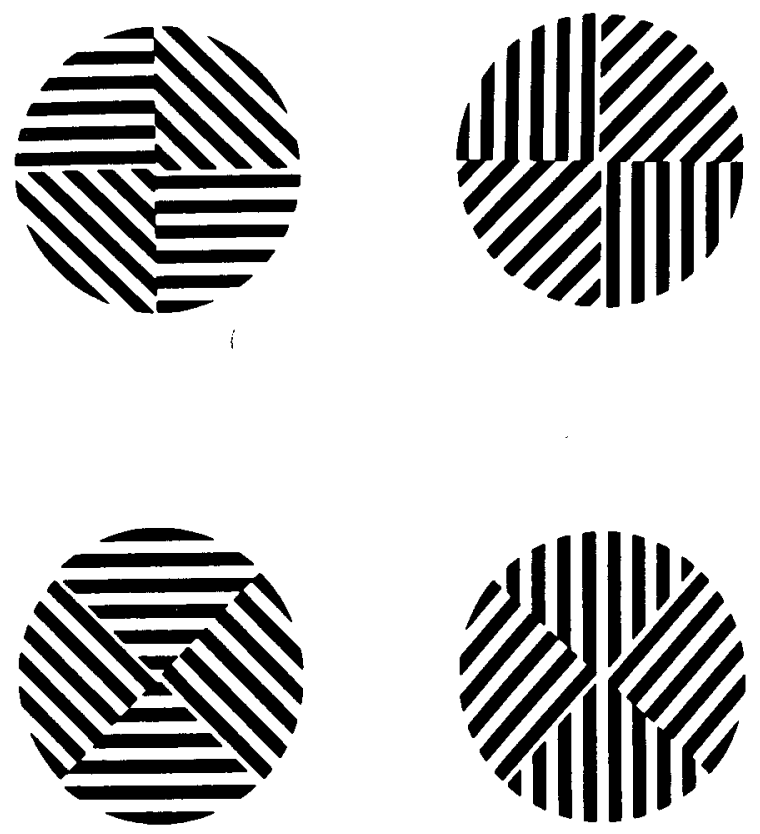

Figure 5. The test patterns used in Experiment 2.

As in Experiment 1, there were more reports to induced than to noninduced patterns. There were no inappropriate responses.

\section{Discussion}

The results show that induction with two grating patterns produces reliable PCCAEs in both these patterns and in patterns whose orientation is orthogonal to them. They do not support the argument that reports on noninduced patterns obtained in Experiment 1 arise from spread of effect from the horizontal or vertical orientations present in the bull's-eye or star to one of the grating patterns. Nevertheless, the results of Experiment 1 are still

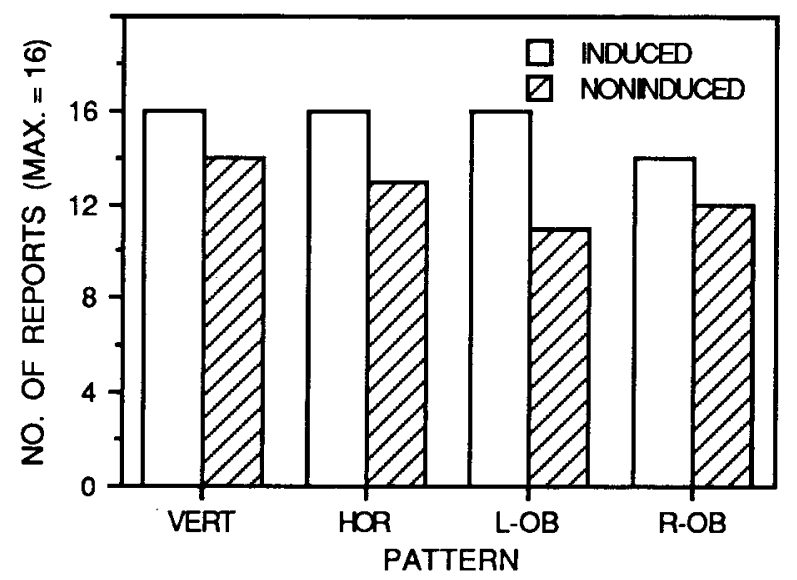

Figure 6. The number of appropriate pattern-contingent color aftereffects reported on induced and noninduced patterns in Experiment 2. VERT $=$ vertical grating. $\mathrm{HOR}=$ horizontal grating. $\mathrm{R}-\mathrm{OB}=$ right oblique grating. $\mathrm{L}-\mathrm{OB}=$ left oblique grating. problematic. It could be argued that reports on noninduced star or bull's-eye segments in Experiment 1 occurred because grating patterns were used, and that only the gratings produce such dual aftereffects. The reports of color aftereffects on noninduced segments of the star and bull'seye patterns in Experiment 1 could be the result of simultaneous color contrast with noninduced grating segments; that is, there may have been a color aftereffect generated on noninduced grating segments of the test patterns used in Experiment 1, but the color reports on the noninduced star and bull's-eye segment are the result of simultaneous color contrast with the grating segments.

Experiment 3 was conducted to see if using only one of the patterns in the induction phase in Figure 1 would produce an aftereffect in both the induced pattern and in its orthogonal patternmate. If after induction with the star alone, for example, reliable aftereffects are reported on both the star and bull's-eye, then this would argue against an interpretation of Experiment 1 in terms of simultaneous contrast with noninduced grating segments only. Rather, such a result would support the notion that orthogonality of the patterns, rather than local elements, determined the aftereffect.

\section{EXPERIMENT 3}

\section{Method}

Subjects. Sixteen undergraduates, naive with respect to the purpose of the experiment, served as subjects. All had normal or corrected-to-normal vision and no known color vision deficiency. During induction, the subjects saw only one of the patterns of Figure 1; 4 subjects were induced with each of the patterns. An additional subject reported seeing colors on the pretest and was not included.

Apparatus and Stimuli. The patterns shown in Figure 1 served as inducing stimuli. They were presented as before. The test patterns, examples of which are shown in Figure 7, consisted of a center-surround arrangement. The surrounding annulus was constructed from one of the inducing patterns. Half of the bipartite central portion of the test pattern was always the pattern orthogonal to the surrounding annulus, whereas the other half was a segment from one of the remaining patterns in Figure 1.

Procedure. As in the previous experiments, each subject was tested in a three-stage procedure of pretest, induction, and posttest. During induction, only one of the patterns shown in Figure 1 was presented in red or green; it was alternated with a homogeneous field in the complementary color. During induction, the pattern and homogeneous field were presented alternately for $10 \mathrm{sec}$, with an intervening $1-\mathrm{sec}$ blank period, for a total time of $20 \mathrm{~min}$. Other details of the procedure were the same as before.

During the posttest, each subject was tested with two patterns, both having the same annular surround corresponding to the induction pattern. The subjects were shown the two test patterns in succession at the appropriate distance and were told to fixate the center of each one in turn. The subjects were instructed to note any areas that seemed pink (or reddish) or green by outlining, as accurately as possible, the appropriate "colored" area on photocopies of the corresponding test patterns and writing down the name of the color.

\section{Results}

If a subject outlined completely any area specific to one of the three patterns in a single test pattern, this was scored 

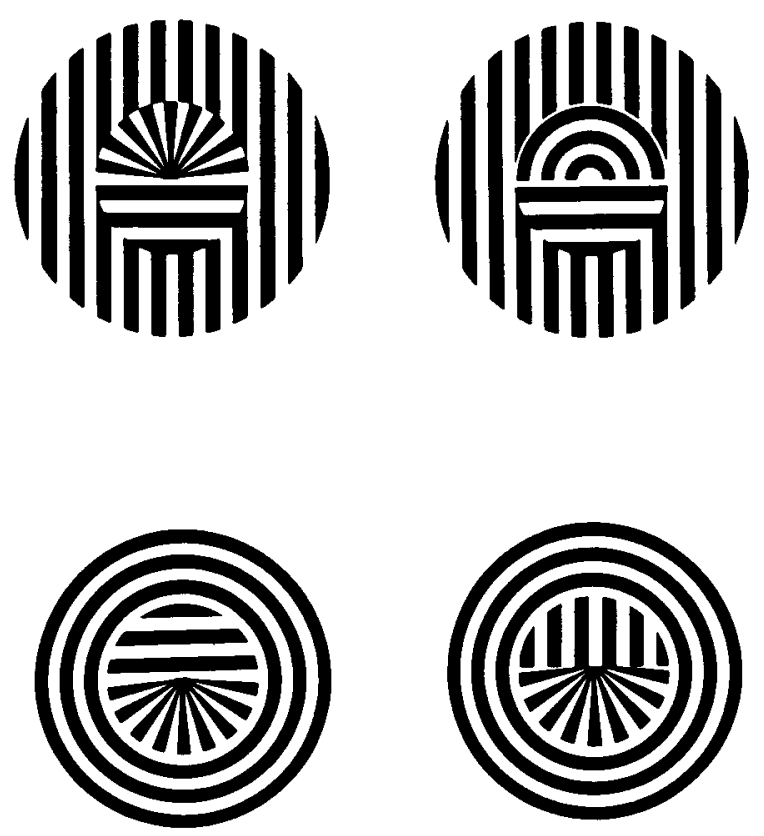

Figure 7. Examples of the test patterns used in Experiment 3.

as a complete PCCAE. Any response outlining a smaller area of any pattern part was labeled a partial PCCAE. As before, inappropriate responses were subtracted from appropriate responses for each subject.

Figure 8 shows the number of complete aftereffects reported on the annular surround of the test pattern corresponding to the four induction conditions. There were no inappropriate complete reports on induced patterns. Figure 9 illustrates the number of complete reports on orthogonal, noninduced patterns. There were no inappropriate complete reports on noninduced pattern segments when these segments were orthogonal to the induction pattern; that is, all such complete color reports were complementary to the induced surrounding annulus. As can be seen from the two figures, there is strong evidence that induction with a single pattern gives rise to an aftereffect both in the induced pattern and in its orthogonal patternmate.

There were a few reports of complete aftereffects to nonorthogonal, noninduced patterns. After induction with the vertical or horizontal grating, only 3 out of a possible $16(19 \%)$ complete reports on the bull's-eye or star segments were obtained. After induction with the bull's-eye or star, 3 complete reports to the horizontal or vertical grating segments were obtained. Thus it appears that complete reports are most likely to be found on patterns that are orthogonal to the induced pattern.

At another level of response, the relation between channels was asymmetric. There were 10 out of 16 possible (63\%) partial reports to the bull's-eye or star segments after induction with the horizontal or vertical gratings and only 3 partial reports to the gratings after induction with the bull's-eye or the star. Qualitative examination of these partial reports also suggested the importance of orthogonality in eliciting aftereffects in noninduced patterns. Most frequently, a subject would outline the roughly orthogonal section of a nonorthogonal pattern segment and report this area as having the same color as the full orthogonal segment. For example, if a subject had been induced with a green vertical grating and later viewed a test pattern composed of the vertical grating in the surround and segments of the horizontal grating and bull's-eye in the center, the horizontal grating would be reported as green and the roughly horizontal section of the bull's-eye would also be reported as green. The remaining section of the bull's-eye containing oblique and vertical orientations might be reported as white or, perhaps, as in the present example, as pink, the same color as the surround. Nine of the 10 partial reports obtained after induction with the gratings were of this form. Two of the three partial reports to nonorthogonal patterns after induction with the bull's-eye or the star could be interpreted in a similar manner.

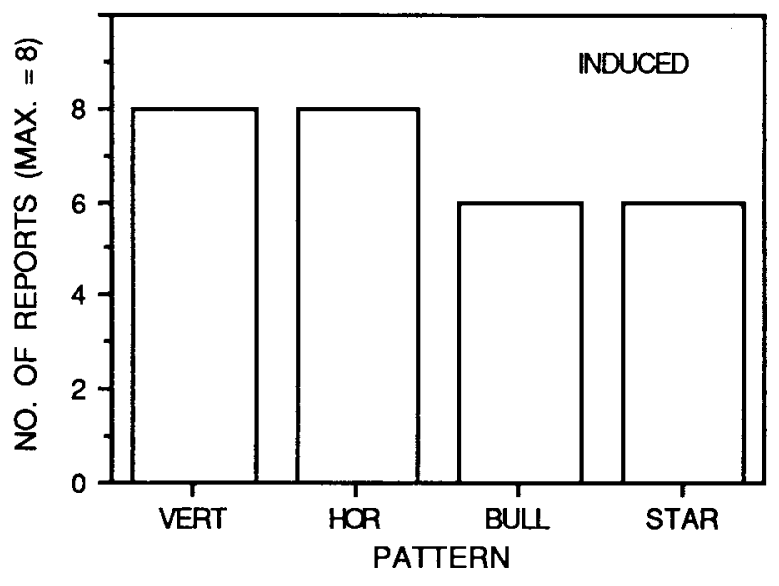

Figure 8. The number of appropriate pattern-contingent color aftereffects reported on induced patterns in Experiment 3.

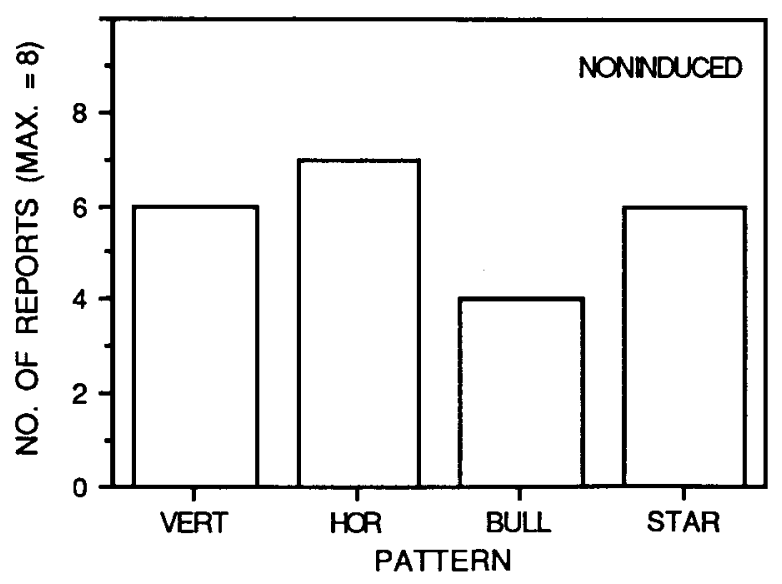

Figure 9. The number of appropriate pattern-contingent color aftereffects reported on noninduced patterns in Experiment 3. 


\section{Discussion}

The results show that two aftereffects are generated by induction with any of the patterns shown in Figure 1. This counters the suggestion made above that only grating patterns are effective in producing a color aftereffect both in themselves and in their orthogonal patternmates. The present results show the gratings to be effective in this way; however, induction with only the bull's-eye or the star also led to a high frequency of appropriate complete aftereffect reports to the noninduced orthogonal patternmate. In other words, complete PCCAEs on noninduced patterns appeared only on patterns orthogonal to an induced pattern.

Partial reports were most frequently reported to nonorthogonal patterns, and the nature of these reports tended to confirm the importance of orthogonality in the generation of aftereffects in noninduced patterns. Areas were outlined that were roughly orthogonal to the induced patterns, and were reported in the color complementary to that seen in the induced pattern.

To support further our interpretation of the partial reports, we conducted another experiment in which only grating patterns were employed. If subjects were giving partial reports only to orthogonal sections of patterns, then there should be few partial reports if the whole of a test pattern segment is not orthogonal to the induction pattern. The subjects were induced with horizontal or vertical gratings and tested with patterns like those used in Experiment 3 , except that the nonorthogonal segments were oblique gratings.

\section{EXPERIMENT 4}

\section{Method}

Subjects. Sixteen undergraduates, naive with respect to the purpose of the experiment, served as subjects. All had normal or corrected-to-normal vision and no known color vision deficiency. An equal number of subjects was assigned to each color-pattern pairing.

Apparatus and Stimuli. The horizontal and vertical grating patterns shown in Figure 1 served as inducing stimuli. They were presented using the same apparatus used in the previous experiments. The test patterns, examples of which are shown in Figure 10, consisted of a surrounding annulus constructed from one of the inducing patterns and a bipartite center. Half of the center was composed of a grating pattern orthogonal to the annular surround, and half was a right or left oblique grating.
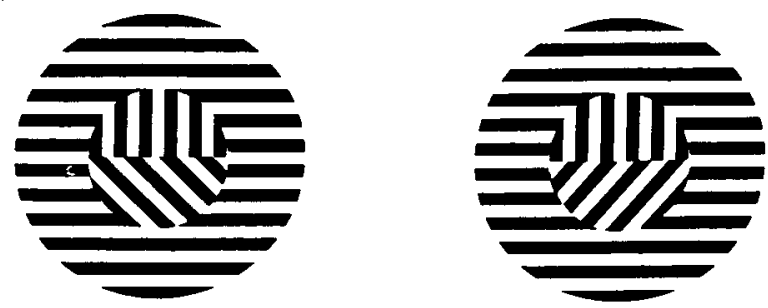

Figure 10. Examples of the test patterns used in Experiments 4, 5 , and 6.
Procedure. Testing was carried out in the same three-stage procedure as before. During induction, a vertical or horizontal grating in red or green was alternated with a homogeneous field in the complementary color for a total time of $20 \mathrm{~min}$. Other procedural details are the same as those in previous experiments.

During the posttest, each subject was tested with two patterns. The annular surround for both test patterns corresponded to the induction pattern. The central portion of one test pattern was composed of a segment of the grating pattern orthogonal to the inducing pattern and a right oblique grating; the other test pattern was the same except that it contained a left oblique grating. As in Experiment 3 , the subjects were instructed to outline any areas on the test patterns that appeared to be colored and label the color of the area pink (or reddish) or green.

\section{Results}

Induction with the gratings was successful in producing an appropriate report in the annular surround in 32 out of 32 possible reports. Fewer aftereffect reports were given on the central orthogonal pattern. For subjects induced with the vertical grating, 10 out of 16 possible reports $(63 \%)$ on the central horizontal segments were appropriate, and there were no inappropriate responses. Also, for subjects induced with the horizontal grating, 10 out of 16 reports on the central vertical segments were appropriate, and there were no inappropriate reports.

There were very few reports of color on the oblique grating. One out of a possible 16 was reported by a subject induced with the vertical grating and 2 by a single subject induced with the horizontal grating. There were no partial PCCAE reports.

\section{Discussion}

The results demonstrate that the probability of reporting an aftereffect on a noninduced pattern depends on the orientation relation between that pattern and the inducing pattern. If induced with a grating pattern, PCCAE reports are reliably obtained to another grating pattern that differs from the orientation of the induction pattern by $90^{\circ}$, but not to a grating pattern that differs by $45^{\circ}$. This is not to say, however, that there is no aftereffect on a grating pattern that is not precisely orthogonal to the induced pattern.

Stromeyer (1984) induced a PCCAE by presenting subjects with a green vertical grating alternating with a uniform magenta field. Following induction, subjects were tested with a pattern composed of two vertical gratings flanking a central grating that was varied in orientation away from the vertical to the horizontal in $22.5^{\circ}$ steps. Measurable aftereffects were found in gratings oriented $45^{\circ}, 67.5^{\circ}$, and $90^{\circ}$ from the flanking vertical gratings. There was, however, an indication that the aftereffect was strongest at the horizontal, that is, orthogonal to the orientation of the induction grating (see Stromeyer, 1984, p. 522, Figure 30.8 ). In our test patterns, subjects were asked simply to name and outline any colors they saw. The PCCAE on orthogonal gratings, which presumably was stronger than PCCAEs on the obliques, was reported most frequently. Furthermore, in our experiment, grat- 
ings at both $45^{\circ}$ to the induced orientation and orthogonal to the induced orientation were present in the test pattern. Stromeyer (1984; see also Webster, Crassini, \& Willenberg, 1987) presented only a single orientation at a time in the center of his test pattern. If the orthogonal test pattern produces the strongest aftereffects, then the oblique pattern in our test patterns could, by contrast, appear white.

Stromeyer (1984) and Webster et al. (1987) also examined the role of simultaneous color contrast in the production of McCollough effects. Color contrast certainly increases the likelihood of reporting PCCAEs (Humphrey et al., 1985). For example, a grating that appears to be achromatic when seen by itself can be made to appear colored if it is flanked by gratings in which a McCollough effect has been induced, or by gratings that are actually colored with lights similar to aftereffect induced colors (Stromeyer, 1984).

All of the test patterns in the present experiments were constructed so that they would have potential color contrast and thus enhance the chances that subjects would detect aftereffects (Humphrey et al., 1985). There is an important difference, however, between Experiments 1 and 2 , and Experiments 3 and 4 . In the latter two experiments, the surrounding annulus in the test pattern was always the inducing pattern. Thus, it could appear that the aftereffect on noninduced patterns is the result of simultaneous contrast with the induced surrounding pattern. This interpretation is not tenable, however, as only the orthogonal portion of the noninduced test patterns reliably led to PCCAE reports. Presumably, if simultaneous contrast were the operative factor, the aftereffects should not have been so dependent on orientation. Furthermore, the results of Experiments 1 and 2 argue against the importance of simultaneous contrast as the major determinant of the aftereffects on noninduced patterns. In both of these experiments, test patterns for noninduced patterns were composed only of parts of noninduced patterns. If PCCAE reports on noninduced patterns depended on simultaneous contrast with surrounding or flanking induced pattern segments in test patterns, then few PCCAE reports should have been obtained. The large number of PCCAE reports runs counter to this interpretation. It appears, then, that reports on noninduced patterns cannot be explained as a simple result of simultaneous contrast with adjacent induced patterns, although color contrast clearly plays an important role in PCCAE reporting.

\section{EXPERIMENT 5}

The first four experiments clearly demonstrated that PCCAEs on noninduced patterns can be reliably established. These experiments all involved presenting complementary colors, red and green, during induction; either patterned stimuli in red and green were presented during induction (Experiments 1 and 2), or a colored pattern was alternated with a homogeneous field in the complementary color (Experiments 3 and 4). The presence of the homogeneous field may help to maintain the saturation of the pattern color and neutralize the afterimages from the pattern color, as suggested by Stromeyer (1984). However, research by Ambler and Foreit (1978) suggests that presentation of a complementary homogeneous field actually assists in the production of an aftereffect on the noninduced, orthogonal pattern. They found that when subjects were adapted to a magenta grating that alternated with an unpatterned green field, there was a trend for reporting an aftereffect on the noninduced orthogonal grating. This did not occur when subjects were adapted to a magenta grating on its own. Although this trend just missed the conventional level of statistical significance, it suggests that the complementary, homogeneous field may play an important role in the production of aftereffects on noninduced patterns. In the next experiment, we examined this possibility by varying the color of the homogeneous field.

\section{Method}

Subjects. Thirty-two undergraduates, naive with respect to the purpose of the experiment, served as subjects. All had normal or corrected-to-normal vision and no known color vision deficiency. Subjects were assigned randomly to the four main conditions of the experiment. One additional subject reported seeing colors on the pretest and was not included.

Apparatus and Stimuli. The horizontal and vertical grating patterns shown in Figure 1 served as inducing stimuli. They were presented using the same apparatus used in the previous experiments. The test pattern used was the same as that shown on the left in Figure 10. A blue Cinemoid filter with a peak transmittance at approximately $460 \mathrm{~nm}$ and an approximate half-width of $30 \mathrm{~nm}$ was also used.

Design. Four different homogeneous fields were used to create four different induction conditions. The grating patterns were always in red or green, but the unpatterned fields were in a color complementary to the grating pattern, the same color as the grating pattern, blue, or blank (black). Half the subjects in each group saw the horizontal grating during induction, and the other half saw the vertical. Equal numbers in each group had red and green as the inducing color.

Procedure. Testing was carried out in the same three-stage procedure as before. During induction, a vertical or horizontal grating in red or green was alternated with one of the homogeneous fields for a total of $20 \mathrm{~min}$. Other procedural details are the same as those in previous experiments.

During the posttest, each subject was tested with one pattern like that shown on the left in Figure 10. The annular surround corresponded to the induction pattern. The central portion of the test pattern was composed of a segment of the grating orthogonal to the inducing pattern and a right oblique grating segment. Because no partial PCCAE reports were obtained in Experiment 4, the subjects were instructed to label any of the three pattern segments that appeared colored. If any two areas appeared to be the same color, the subjects were asked to indicate which area appeared more vivid or intense.

\section{Results}

Induction with the gratings was successful in producing an appropriate color report in $\mathbf{3 0}$ out of 32 possible reports. Figure 11 shows the number of appropriate reports for the induced and noninduced orthogonal patterns, as well as the number of color reports to the oblique 

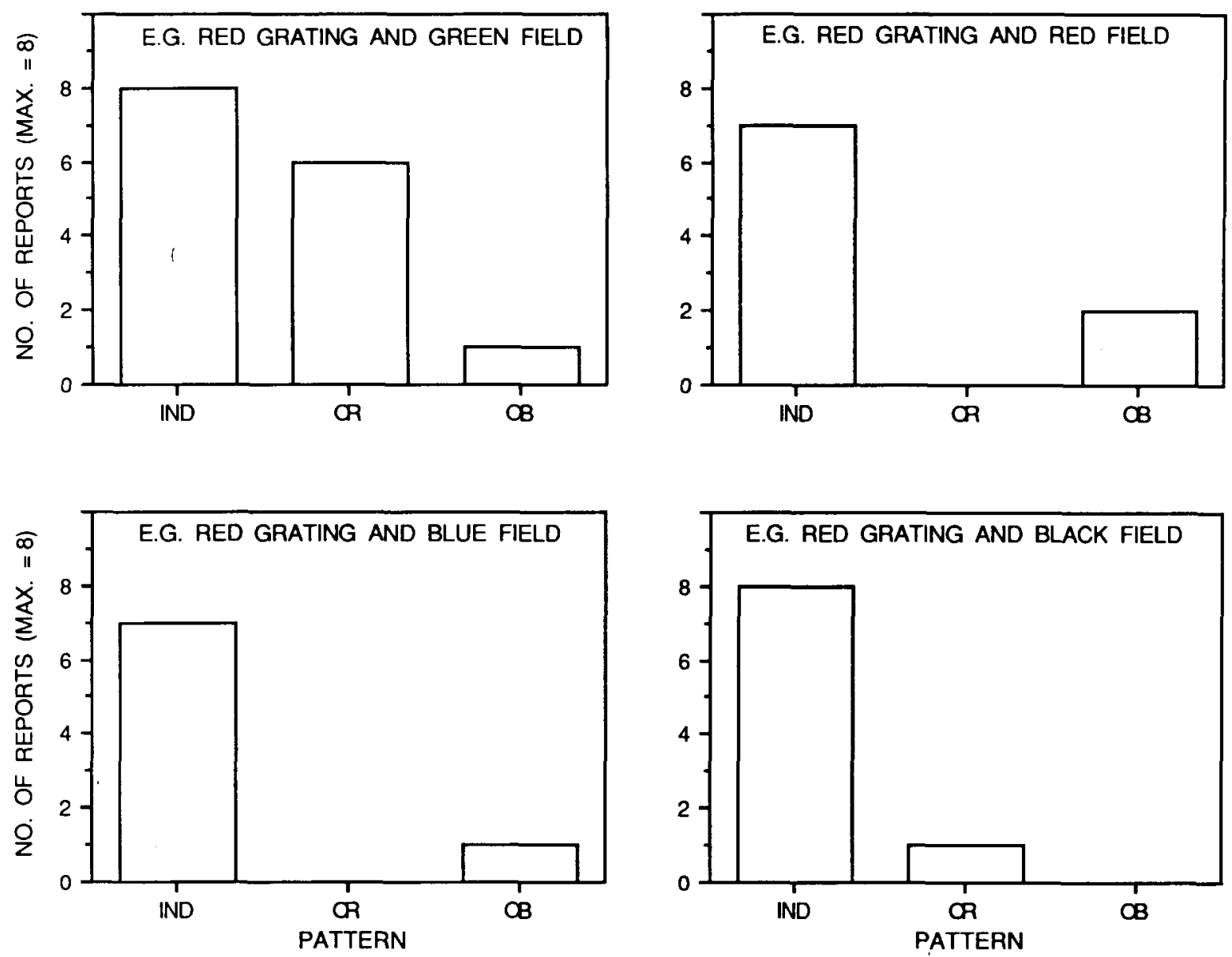

Figure 11. The number of appropriate pattern-contingent color aftereffects obtained in the four induction conditions of Experiment 5. IND = induced. OR = orthogonal noninduced. $O B=$ oblique noninduced.

pattern. There were only 4 reports to the oblique pattern, and only the single report in the condition with the blue homogeneous field was the same color as the surround. One subject in the condition induced with complementary colors reported the same color (appropriate) on the orthogonal and oblique pattern segments, but indicated that the orthogonal grating was more intense. It is clear from Figure 11 that only the condition with the homogeneous field in a color complementary to the color of the inducing pattern reliably produced color reports on the noninduced orthogonal pattern.

\section{Discussion}

The results for the condition involving complementary colors during induction replicated the results of Experiment 4 . Appropriate colors were consistently reported to the noninduced orthogonal grating, but not to the oblique grating. The other three test conditions produced few color reports.

The results are in agreement with the trend noted by Ambler and Foreit (1978): a condition in which induction with a colored grating pattern was alternated with an unpatterned field in complementary color tended to produce color reports on a noninduced orthogonal grating, whereas another condition in which the colored grating was not alternated with any color did not show such a trend. In addition, there was a lack of color reports on noninduced patterns if a homogeneous field in the same color as the inducing pattern or a color not complementary to the inducing pattern (i.e., blue) was alternated with a colored pattern during induction. The condition involving the blank field did not replicate the results of Stromeyer (1969), who found that such an arrangement produced aftereffects on the noninduced patterns. It is not clear why Stromeyer (1969) obtained these results, but it should be noted that Ambler and Foreit (1978) also failed to replicate them.

It could be objected that the only condition that maintained the color saturation of the pattern and led to a strong aftereffect in the induced pattern was one in which it was alternated with an unpatterned field in a complementary color. Other induction conditions could lead to a weaker aftereffect on the induced pattern. Ambler and Foreit (1978) did in fact find that the aftereffect on induced patterns was stronger in conditions involving complementary colors than in those involving a single color. Thus 
the strength of the aftereffect in the surround of the test patterns used in Experiment 5 might have influenced the likelihood that aftereffects would be reported in the noninduced patterns. If the aftereffect strength were greatest in the condition involving complementary colors, this could lead to the higher level of color reports on the noninduced patterns.

Although the preceding interpretation of the results is plausible, there are some problems with it. First, at least according to our measures, all conditions produced a high level of appropriate color reports on the induced patterns. Admittedly, our measure of color naming does not lead to fine quantitative differentiation; however, within its limits, there do not appear to be differences between the conditions. Second, the blue unpatterned field should have been quite effective in maintaining color saturation, but this condition did not lead to a high level of color reports on the noninduced patterns. Finally, the trend noted by Ambler and Foreit (1978) occurred with test patterns in which only the noninduced pattern was shown; thus their results were not due to simultaneous color contrast.

\section{EXPERIMENT 6}

The results of Experiments 3, 4, and 5 and the earlier research of Ambler and Foreit (1978) demonstrate that an appropriately colored patternless field can be substituted for a colored grating and produce an aftereffect in a pattern orthogonal to the pattern seen during induction. Experiment 5 suggests that to obtain such aftereffects on noninduced patterns, two roughly complementary colors must be used during induction. However, Stromeyer (1969) found that alternating one colored grating with another orthogonally oriented achromatic grating also produced aftereffects on both gratings. For example, induction with a red vertical grating that alternated with an achromatic horizontal grating produced a green aftereffect on the vertical grating and a red aftereffect on the horizontal grating. Such results suggest that complementary colors are not necessary to produce aftereffects on noninduced patterns, but it may be that "complementary" (i.e., orthogonal) patterns are necessary. The following experiment examined the production of aftereffects on noninduced patterns using three different induction conditions: a colored vertical grating was alternated with achromatic horizontal, achromatic oblique, or achromatic vertical gratings.

\section{Method}

Subjects. Twenty-four undergraduates, naive with respect to the purpose of the experiment, were subjects. All had normal or corrected-to-normal vision and no known color vision deficiency. Subjects were assigned randomly to the three conditions of the experiment.

Apparatus and Stimuli. Horizontal, vertical, and oblique gratings such as those used in the previous experiments were the inducing stimuli. They were presented in the same apparatus and manner as in the previous experiments. The test pattern was the same as that used in Experiment 5.
Design. Three different two-pattern pairings were used during induction. One pattern was always in red or green, whereas the other was in black and white. One group saw orthogonal patterns during induction, another group saw patterns of identical orientation during induction (i.e., both gratings were vertical or both gratings were horizontal), and a third group saw a colored vertical or horizontal grating alternated with an achromatic right oblique grating. Half of the subjects in each group saw a colored (either red or green) vertical grating during induction, whereas the other half saw a colored (red or green) horizontal grating.

Procedure. Testing was carried out in the same three-stage procedure as before. During induction, a vertical or horizontal grating in red or green was alternated with a black-and-white grating for a total of $20 \mathrm{~min}$. Other procedural details were the same as in the previous experiments.

During the posttest, each subject was tested with one pattern. The annular surround corresponded to the induction pattern. The central portion of the test pattern was composed of a segment of the grating orthogonal to the inducing pattern and a right oblique grating segment. The subjects were instructed to label any of the three pattern segments that appeared colored. If any two areas appeared to be the same color, the subjects were asked to indicate which area appeared more vivid or intense.

\section{Results}

Figure 12 presents the results for two of the three induction conditions. As can be seen, these two conditions produced different patterns of responding on both the induced and noninduced patterns. If the grating patterns were orthogonal, a high number of PCCAE reports were made both to the pattern presented in color during induction and to the pattern presented in black and white. Similarly, if a colored vertical or horizontal grating was alternated with an achromatic oblique grating, a high number of reported aftereffects were noted on the induced vertical or horizontal portion of the test pattern and on the oblique section. If, however, a colored vertical (or horizontal) pattern was alternated with a black-and-white vertical (or horizontal) pattern during induction, no aftereffects were reported on any of the test pattern segments.

\section{Discussion}

The condition in which orthogonal patterns were alternated during induction replicated the finding of Stromeyer (1969) that a colored grating that alternated with an orthogonal black-and-white grating produces appropriately colored aftereffects on both horizontal and vertical gratings. The present results suggest that reporting of such aftereffects is limited to the orientations seen during induction. Induction with horizontal and vertical gratings did not result in color reports to the oblique grating. In the condition involving a colored vertical or horizontal grating that alternated with a black-and-white oblique grating, a high number of reports were made to the pattern seen in color during induction, as well as to the oblique grating, but only one report was made to the pattern that was orthogonal to the colored pattern seen during induction. Finally, if the orientation of the colored pattern and achromatic pattern were the same during induction, no aftereffects were reported on any segments of the test pattern. 

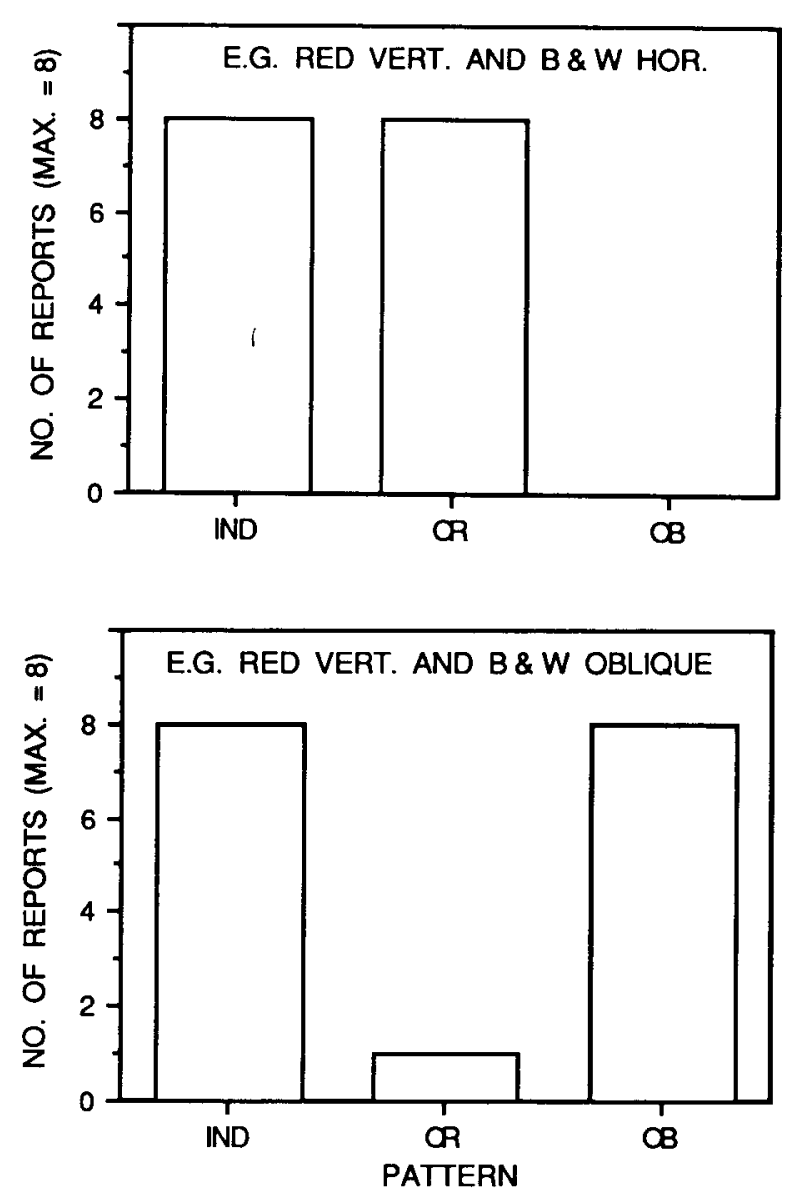

Figure 12. The number of appropriate pattern-contingent color aftereffects reported in two of the three induction conditions of Experiment 6 . The condition in which, for example, a red vertical grating was alternated with a black-and-white vertical grating during induction is not displayed because there were no aftereffect reports to any of the test pattern segments.

The explanation that most simply accounts for our results is the one offered by Murch and Hirsch (1972) to account for Stromeyer's (1969) results. They argued that the viewing of, for example, a red vertical grating during induction would fatigue retinal receptors sensitive to red; as a result, the white background of the horizontal grating was processed by receptors most sensitive to blue and green. Thus the induction condition produced an effective blue-green horizontal grating that alternated with a red vertical grating. Murch and Hirsch supported their notion that colored afterimages were effective in inducing PCCAEs by producing aftereffects as a result of superimposing colored afterimages on black-and-white vertical and horizontal gratings.

The interpretation offered by Murch and Hirsch (1972) readily accounts for the results of our Experiment 6. During induction, the achromatic pattern would be "colored" in a complementary way by the afterimage produced as a result of fixating the colored pattern. If the two patterns seen during induction are of different orientation (differ- ent by more than about $20^{\circ}$; Stromeyer, 1978), aftereffects are readily established in both patterns. If the patterns are the same orientation, then this means that the pattern is presented in two approximately complementary colors and, as a result, no aftereffects are produced.

\section{GENERAL DISCUSSION}

This series of experiments demonstrates that PCCAEs can be produced reliably on patterns that were never used during induction, provided that certain conditions are met. Generally, noninduced patterns on which aftereffects are reported are orthogonal to the patterns used during induction. We will discuss later why this should be so. The aftereffects on noninduced patterns are reported reliably only if complementary colors are used in the induction phase, suggesting that the presence of both colors is important, if not crucial, in the formation of the aftereffects (see also Ambler \& Foreit, 1978; Humphrey et al., 1985).

Our model for aftereffects on noninduced patterns is based on the notion that inspection of a particular pattern during induction reduces the sensitivity of the coding mechanism for that pattern, a mechanism that is specifically tuned to certain orientational features of pattern contours. With stimulus offset, the balance of activity in different coding mechanisms shifts away from that that occurred during inspection of the pattern, so that the level of activity of mechanisms coding for contrasting features predominates. The maximum contrast in orientation coding occurs for orientations approximately perpendicular to the inducing pattern. This new level of activity is associated with color that is present following the inducing pattern.

Parenthetically, we should note that there are other indications of such opponent processing of orthogonal orientations. MacKay $(1957,1961)$ demonstrated that highfrequency radial, concentric circle, and grating-like patterns induce spatially complementary effects. Campbell and Maffei (1971) showed that adaptation to a grating near the vertical produces a tilt aftereffect for test gratings near the horizontal. Furthermore, Heeley (1979) presented evidence that a spatial frequency shift can be obtained at test orientations that are orthogonal to the orientation of the adapting stimulus.

Our explanation is similar in some respects to the one offered to account for the results of Experiment 6, and also for experiments reported in Murch and Hirsch (1972). Their experiments involved pairing a black-and-white pattern with a colored afterimage, which then served to set up a PCCAE. We now propose that a shift in the neural activity that mediates contour coding is also sufficient to produce a PCCAE when associated with color, even though no second patterned stimulus is presented. It seems that the hypothesized shift in coding activity, combined with a complementary negative afterimage, is not in itself sufficient to generate a PCCAE. If it were, we should expect to find such an aftereffect in Experiment 5; specifically, this should occur in the condition in which a 
colored pattern was alternated with a black, colorless field. In fact, the evidence denies this (see also Ambler \& Foreit, 1978). It is possible, however, that the use of the black field may have weakened the strength of the afterimage. Perhaps if we had used a white intervening field, we would have obtained different results because of the production of more obvious afterimages.

The condition in Experiment 5 in which a red colored pattern was alternated with a red homogeneous field would not be expected to produce aftereffects on noninduced patterns according to our hypothesis. We also found in other research that presenting two patterns in the same color during induction led to very few reports on induced or noninduced patterns (Humphrey et al., 1985), so it is not surprising that we did not obtain such reports in Experiment 5 . One possible reason for such low levels of reported effects is a lack of color contrast in the test stimuli, but the more likely explanation is that the presence of the same color in both phases of induction leads to an induction regime in which a pattern and the hypothesized shift in coding are both associated with the same color. The condition involving a blue homogeneous field in Experiment 5 may not have resulted in aftereffects because this color generally produces very weak effects, even when presented on a visible grating (Fidell, 1968; Stromeyer, 1969).

Our concept of how noninduced aftereffects are generated implies that a color can be associated both with the hypothesized shift in coding activity and with the presentation of a real pattern. In Experiments 1 and 2, two nonorthogonal patterns in complementary colors were presented during induction. Reliable PCCAEs were generated both on the induced pattern and on the noninduced pattern. According to the present account, the condition that involves, for example, a red radial pattern and a green vertical grating (Experiment 1) during induction would be explained as follows: Following inspection of the red radial pattern, dominant neural activity shifts to the coding mechanism for perpendicular orientations (i.e., concentric circles), which are now associated with the color on the vertical grating, namely green. Similarly, after inspecting the vertical grating, an orthogonal shift in dominant coding activity becomes associated with the red color of the radial pattern. Our earlier research (Emerson et al., 1985; Humphrey et al., 1985) demonstrated that separate PCCAEs can be established in a single observer for each of the patterns of Figure 1 with relatively little interference. Thus it is conceivable that the hypothesized change in dominant coding activity following presentation of an inducing pattern, as well as a visually present pattern in the second phase of the induction sequence, both become associated with a single color without too much interference.

There is some evidence (Fidell, 1970) that the strongest PCCAEs are generated when the patterns used in induction approach orthogonal orientations, each shown in two complementary colored lights. One of us has shown elsewhere (Dodwell \& O'Shea, 1987) that orthogonality is not sufficient on its own. Local orthogonality does not generate PCCAEs; one requires global patterns to produce strong effects. Thus, when we propose that orthogonal coding activities are selectively desensitized or become dominant, we do not have in mind that these would be simple feature detectors of the type popularly supposed to underlie the McCollough effect. Not only is the time course for fatiguing of such detectors far too short to be a useful model for these effects, it is also necessary that the patterns that generate PCCAEs have some global structure. It is therefore plausible to postulate that the neural mechanisms involved are at a higher level than is commonly supposed, and we suggest again that the vectorfields of LTG/NP have the requisite properties to account for the phenomena described in this paper (see Dodwell, 1984; Emerson et al., 1985).

Recently, Grossberg (1987) has proposed a longterm-memory (LTM) trace within the feature-detection pathways, which might be taken to discount our claim that the time course of color aftereffects is too long to be taking place at the simple feature-detection level. Two points can be made. First, Grossberg defined the LTM trace as a network characteristic, not as a simple detector property. This makes our point. Second, our evidence shows that the networks involved probably have vectorfield properties. If the PCCAEs are global in nature, it seems reasonable to postulate the neural mechanisms that mediate the global effects-vectorfields in our view-are where the contingent color biasing occurs. We do not currently have independent evidence on the time-course of color-biasing in cortical vectorfields, so this notion, like Grossberg's, is conjectural at present.

The pairs of patterns shown in Figure 1 represent four of the "primitive" patterns of LTG/NP (Dodwell, 1983), which are based on the orbits generated by operators that have the local orthogonal property required to form a Lie algebra. The discovery that pattern coherence, or global organization, is important in the inducing pattern (Dodwell \& O'Shea, 1987) adds strong support to the postulate that PCCAEs are in fact generated at the level of vectorfields as described in the LTG/NP model. It is interesting to note that the principle of transverse control in that model (Hoffman \& Dodwell, 1985) implies networks of essentially orthogonal elements that both generate and control the extent of visual contours.

Having invoked LTG/NP as a framework within which to account for PCCAEs in general, and for the effects on noninduced orthogonal patterns in particular, we end by noting that the LTG/NP model does not have an explanation, as it stands, for why PCCAEs occur in the first place. There is, however, no other reasonable postulate in the literature of a useful role for them in vision either. It is also true that LTG/NP does not give an adequate computational account of the genesis of PCCAEs. These are matters that we shall address in subsequent publications.

\section{REFERENCES}

Ambler, B. A., \& Foreit, K. G. (1978). Induction of the McCollough effect: II. Two different mechanisms. Perception \& Psychophysics, 24, 466-470. 
CAmpbell, F. W., \& MAFFeI, L. (1971). The tilt after-effect: A fresh look. Vision Research, 11, 833-840.

DODWELL, P. C. (1983). The Lie transformation group model of visual perception. Perception \& Psychophysics, 34, 1-16.

DoDWELL, P. C. (1984). Local and global factors in figural synthesis. In P. C. Dodwell \& T. M. Caelli (Eds.), Figural synthesis (pp. 219. 248). Hillsdale, NJ: Erlbaum.

DODWELl, P. C., \& O'SheA, R. P. (1987). Global factors generate the McCollough effect. Vision Research, 27, 569-580.

Emerson, V. F., Humphrey, G. K., DodWell, P. C. (1985). Colored aftereffects contingent on patterns generated by Lie transformation groups. Perception \& Psychophysics, 37, 155-162.

FIDELL, L. S. (1968). Pattern-specific complementary hue aftereffects: More on the McCollough phenomenon. Unpublished doctoral dissertation, University of Michigan, Ann Arbor.

FIDELL, L. S. (1970). Orientation specificity in chromatic adaptation of human "edge-detectors." Perception \& Psychophysics, 8, 235-237.

GrossBerg, S. (1987). Cortical dynamics of three-dimensional form, color, and brightness: II. Binocular theory. Perception \& Psychophysics, 41, 117-158.

HARRIS, C. S. (1980). Insight or out of sight? Two examples of perceptual plasticity in the human adult. In C. S. Harris (Ed.), Visual coding and adaptability (pp. 95-149). Hillsdale, NJ: Erlbaum.

Heeley, D. W. (1979). A perceived spatial frequency shift at orientations orthogonal to adapting gratings. Vision Research, 19, 1229-1236.

HofFmaN, W. C. (1966). The Lie algebra of visual perception. Journal of Mathematical Psychology, 3, 65-98. (Errata; ibid, 1967, 4, 348-349).

HoffmaN, W. C. (1978). The Lie transformation group approach to visual neuropsychology. In E. L. J. Leeuwenbert \& H. Buffart (Eds.), Formal theories of visual perception (pp. 27-66). Chichester, England: Halsted Press.

Hoffman, W. C., Dodwell, P. C. (1985). Geometric psychology generates the visual gestalt. Canadian Joumal of Psychology, 39, 491-528.

Humphrey, G. K., Dodwell, P. C., Emerson, V. F. (1985). The roles of pattern orthogonality and color contrast in the generation of pattern-contingent color aftereffects. Perception \& Psychophysics, 38, 343-353.

MACKAY, D. M. (1957). Moving images produced by regular stationary patterns. Nature, 180, 849-850.

MACKAY, D. M. (1961). Interactive processes in visual perception. In W. A. Rosenblith (Ed.), Sensory communication (pp. 339-355). Cambridge, MA: MIT Press.

McCollough, C. (1965). Color adaptation of edge-detectors in the human visual system. Science, 192, 1115-1116.

Murch, G., Hirsch, J. (1972). The McCollough effect created by complementary afterimages. American Joumal of Psychology, 85. 241-247.

Skowbo, D. (1986). McCollough effects as conditioned responses? Reply to Allan and Siegel. Psychological Bulletin, 100, 394-397.

Stromeyer, C. F. (1969). Further studies of the McCollough effect. Perception \& Psychophysics, 6, 105-110.

Stromeyer, C. F. (1978). Form-color aftereffects in human vision. In R. Held, H. W. Leibowitz, \& H.-L. Teuber (Eds.), Perception: Handbook of sensory physiology (Vol. 8). New York: Springer-Verlag.

STromeYER, C. F. (1984). Orientation-specific color aftereffects and simultaneous color contrast. In L. Spillman \& B. R. Wooten (Eds.), Sensory experience, adaptation and perception (pp. 509-527). Hillsdale, NJ: Erlbaum.

Webster, W. R., Crassini, B., Willenberg, K. (1987). Simultaneous color contrast from McCollough effects is spatially contingent. Perception \& Psychophysics, 41, 402-408.

(Manuscript received January 4, 1988; revision accepted for publication August 9, 1988.) 